\title{
Uso de prototipagem rápida ou manufatura aditiva para estudos de casos clínicos e planejamento de técnica cirúrgica utilizando modelos 3D
}

\author{
Use of rapid prototyping or additive manufacturing for clinical case studies and surgical technique
} planning using 3D models

Uso de prototipos rápidos o fabricación aditiva para estudios de casos clínicos y planificación de técnicas quirúrgicas utilizando modelos 3D

\begin{abstract}
Resumo
O processo de criação tridimensional ou manufatura aditiva tem grande futuro dentro da medicina. A aplicação de sucessivas camadas produz objetos com incrível precisão em relação a peça real. A criação de modelos em 3D possibilita uma visualização mais efetiva de estruturas anatômicas facilitando o ensino-aprendizagem, o estudo préoperatório de cirurgias complexas e até a prática médica com a melhoria da técnica clínica. O presente trabalho demonstra a viabilidade da prototipagem rápida ou impressão digital 3D no processo ensino-aprendizagem na área de anatomia humana estendendo-se entre os campos dos casos clínicos, planejamento cirúrgico e técnicas cirúrgicas. Trata-se de um estudo descritivo, baseado em uma revisão integrativa da literatura, com buscas nas bases de dados PubMed, LILACS, SciELO e Google Acadêmico, utilizando os descritores "Impressão Tridimensional", "Anatomia", "Educação Médica". Muitos estudos mostram benefícios evidentes no processo ensino-aprendizagem em anatomia utilizando modelos 3D produzidos com menor custo e grande precisão. Além da aplicabilidade no ambiente acadêmico e profissional ainda há desafios a serem enfrentados como o custo das impressoras e a capacitação para o uso. Nesse sentido, a aplicabilidade dessa tecnologia possui um futuro promissor não só na medicina que envolvem tanto o campo básico do conhecimento quanto na resolução de problemáticas melhorando a eficiência dos profissionais tanto no planejamento quanto na prática cirúrgica.
\end{abstract}

Palavras-chave: Anatomia; Educação médica; Impressão tridimensional.

\begin{abstract}
The three-dimensional creation process or additive manufacturing has a great future in medicine. Applying successive layers produces objects with incredible precision in relation to the real part. The creation of 3D models enables a more effective visualization of anatomical structures, facilitating teaching-learning, preoperative study of complex surgeries and even medical practice with the improvement of clinical technique. The present work demonstrates the feasibility of rapid prototyping or 3D digital printing in the teaching-learning process in the field of human anatomy, extending between the fields of clinical cases, surgical planning, and surgical techniques. This is a descriptive study, based on an integrative literature review, with searches in the PubMed, LILACS, SciELO and
\end{abstract}


Academic Google databases, using the descriptors "Tridimensional Printing", "Anatomy”, "Medical Education". Many studies show clear benefits in the teaching-learning process in anatomy using 3D models produced with lower cost and great precision. In addition to the applicability in the academic and professional environment, there are still challenges to be faced, such as the cost of printers and training for their use. In this sense, the applicability of this technology has a promising future not only in medicine that involve both the basic field of knowledge and problem solving, improving the efficiency of professionals both in planning and in surgical practice.

Keywords: Anatomy; Medical education; Three-dimensional printing.

\begin{abstract}
Resumen
El proceso de creación tridimensional o fabricación aditiva tiene un gran futuro en la medicina. La aplicación de capas sucesivas produce objetos con una precisión increíble en relación con la pieza real. La creación de modelos 3D permite una visualización más eficaz de las estructuras anatómicas, facilitando la enseñanza-aprendizaje, el estudio preoperatorio de cirugías complejas e incluso la práctica médica con la mejora de la técnica clínica. El presente trabajo demuestra la viabilidad del prototipado rápido o impresión digital 3D en el proceso de enseñanzaaprendizaje en el campo de la anatomía humana, extendiéndose entre los campos de casos clínicos, planificación y técnicas quirúrgicas. Se trata de un estudio descriptivo, basado en una revisión integradora de la literatura, con búsquedas en las bases de datos PubMed, LILACS, SciELO y Academic Google, utilizando los descriptores "Impresión tridimensional", "Anatomía", "Educación médica". Numerosos estudios muestran claros beneficios en el proceso de enseñanza-aprendizaje en anatomía utilizando modelos 3D producidos con menor costo y gran precisión. Además de la aplicabilidad en el entorno académico y profesional, aún quedan desafíos por enfrentar, como el costo de las impresoras y la capacitación para su uso. En este sentido, la aplicabilidad de esta tecnología tiene un futuro prometedor no solo en la medicina que involucra tanto el campo básico del conocimiento como la resolución de problemas, mejorando la eficiencia de los profesionales tanto en la planificación como en la práctica quirúrgica.
\end{abstract}

Palabras clave: Anatomía; Educación médica; Impresión tridimensional.

\title{
1. Introdução
}

A Prototipagem rápida consiste na transformação de imagens digitais em modelos físicos tridimensionais (Provenzano et al., 2020; Krishnasamy et al., 2021). A Manufatura Aditiva (MA) ou mais usualmente conhecida como impressão tridimensional (3D), é um processo de produção de objetos geométricos utilizando materiais (sintéticos ou orgânicos), que consiste na fabricação, a partir de um modelo tridimensional computadorizado, e exteriorizado por meio de impressoras que adicionam sucessivas camadas do material (Lozano et al., 2017; Volpato et al., 2017). Nada mais é do que a adição de camadas de um determinado material, de maneira automatizada que reproduz fisicamente um modelo 3D virtual (Corazza et al., 2020; Silva et al., 2020).

$\mathrm{Na}$ medicina o uso dos exames de imagens em duas dimensões (2D) como o raio $\mathrm{X}$ (RX), a tomografia computadorizada (TC) e a ressonância magnética (RM) revolucionaram o processo diagnóstico e a tomada de decisão, melhorando de forma geral as condutas e o prognóstico (Wilk et al., 2020). Por outro lado, esse tipo de auxílio diagnóstico apresenta algumas limitações como a necessidade de uma boa compreensão das imagens em duas dimensões, conhecimento da anatomia humana e sua apresentação real e patológica (Tack et al., 2016; Guimarães et al. 2017; Hecht-López \& LarrazábalMiranda, 2018; Wu et al., 2018; Soares Neto et al., 2020).

Com o desenvolvimento de novas tecnologias que transformam imagens e modelos computadorizados em objetos reais tridimensionais, a percepção antes limitada a avaliação bidimensional alterou-se. Softwares produzem, por meio da engenharia reversa que converte imagens de exames digitais comuns no dia a dia médico (RX, TC, RM e até ultrassonografias), peças volumétricas (Allen et al., 2015; Araujo et al., 2019). Modelos fotogramétricos são produzidos com uso de scanners que digitalizam objetos como peças anatômicas e permitem a reprodução com a impressão 3D (Jones, 2019; Krishnasamy et al., 2021). Todos esses são meios que facilitam o processo ensino-aprendizagem contínuo de anatomia, fisiologia, casos clínicos, além de auxiliar nos processos cirúrgicos mostrando uma área dentro do campo médico (Singh et al., 
2016; Cramer et al., 2017; Smith et al., 2018).

Com a viabilização da produção das peças em 3D, por meio da prototipagem rápida, com proporções de alta exatidão em relação aos exemplos reais, esse recurso viabiliza a construção de um banco de dados para que professores e estudantes utilizem esse material nas aulas práticas (Louredo et al., 2019; Awadh et al. 2020; Ye et al., 2020). O uso da prototipagem se mostra uma alternativa como solução aos déficits observados como a obtenção de peças cadavéricas e a manutenção desses materiais orgânicos. Considerando os critérios éticos e burocráticos, a aquisição das peças anatômicas é fundamental para o estudo anatômico e clínico de tecidos e órgãos. No entanto, o desafio é a deterioração dos cadáveres, mesmo com diferentes métodos de conservação há algum grau de alteração, visto que as noções de proporcionalidade, volumetria e de outros detalhes anatômicos podem ser perdidos (Lim et al., 2016; Moraes \& Muniz, 2018; Cordeiro \& Menezes, 2019). Além disso, a aquisição de novas peças tem sido um trabalho dificultado devido aos processos de doação de corpos complexos e burocráticos, de acordo com cada legislação local.

Para compreender a anatomia muitas das vezes o estudante precisa de pensamentos abstratos, como uma virtualização nesse processo de entendimento das estruturas, para que os conceitos possam ser percebidos (Balestrini \& Campo-Celaya, 2016). Ainda assim, algumas partes das peças anatômicas permitem visualização limitada ao corte produzido durante a dissecação e dúvidas permanecem nesse processo global, já que o corpo humano é algo tridimensional, considerando o estudo de peças macro e microscópicas. Esse conhecimento limita-se ainda mais quando se estuda os processos de embriogênese (Hecht-López \& Larrazábal-Miranda, 2018; Keenan \& Ben Awadh, 2019).

O estudo dos mais diversos tipos de tecidos e órgãos já é uma realidade. É possível estudar de forma tridimensional, por exemplo, de vasos sanguíneos estenosados, ateroscleróticos, aneurismas e até dissecções com enorme precisão, podendo, assim, determinar a melhor conduta pré-operatória e a avaliação global do caso que antes dependia apenas de exames bidimensionais como a angiografia (Hermsen et al., 2020). Nesse contexto, com o desenvolvimento da impressão digital, um novo passo foi tomado. A facilidade de desenvolver modelos tridimensionais possibilitou recriar modelos reais de patologias, promovendo melhorias no estudo da anatomia e da patologia das mais diversas estruturas e variações anatômicas, além de casos clínicos complexos com a criação de modelos com grande similaridade à realidade, associado a um enriquecimento do processo ensino-aprendizagem (Lozano et al., 2017; Smith et al., 2018; Keenan \& Ben Awadh, 2019; Smith \& Dasgupta, 2020).

De tal modo, novas metodologias que consigam abranger tanto a educação no ambiente acadêmico com o favorecimento dos métodos ativos de aprendizagem, quanto pela interdisciplinaridade entre os diversos campos de atuação dentro da área são focos do processo ensino-aprendizagem (Guimarães et al. 2017; Erolin, 2019; Louredo et al., 2019; Ye et al., 2020; Mendonça et al., 2021). Por conseguinte, este estudo demonstra a viabilidade da prototipagem rápida ou impressão digital 3D no processo ensino-aprendizagem na área de anatomia humana estendendo seu uso entre os campos de estudo dos casos clínicos, planejamento cirúrgico e técnicas cirúrgicas.

\section{Metodologia}

Trata-se de um estudo descritivo, baseado em uma revisão integrativa da literatura, um método de pesquisa que permite a síntese de múltiplos estudos publicados possibilitando conclusões a respeito de uma determinada área (Koche, 2011). Foram utilizadas as seguintes etapas: identificação do tema; seleção da questão de pesquisa; coleta de dados pela busca na literatura, nas bases de dados eletrônicas, com estabelecimento de critérios de inclusão e exclusão para selecionar a amostra; elaboração de um instrumento de coleta de dados com as informações a serem extraídas; avaliação dos estudos incluídos na 
revisão integrativa; interpretação dos resultados e apresentação dos resultados evidenciados.

A questão norteadora da pesquisa foi: "Qual a aplicabilidade da prototipagem rápida na produção de modelos para estudos de anatomia, casos clínicos, planejamento e técnica cirúrgica"? Para responder a tal questionamento, foi executada uma busca nas bases de dados: PubMed (National Library of Medicine and National Institutes of Health), LILACS (Literatura Latino-Americana e do Caribe em Ciências da Saúde), SciELO (Scientific Electronic Library Online) e Google Acadêmico. Foram utilizados os Descritores em Ciências da Saúde (DeCS) "Impressão Tridimensional", "Anatomia", "Educação Médica" em inglês e português com o auxílio de operadores Booleanos (AND/OR). A coleta de dados foi realizada entre maio e julho de 2021. Os critérios de inclusão dos estudos foram: estudos originais, de revisão e relatos que trouxessem dados a respeito da aplicação da prototipagem, artigos disponíveis com texto completo; estudos publicados nos idiomas português, inglês e espanhol, sem restrição de data de publicação. Foram excluídos artigos disponíveis apenas em resumo, artigos de opinião, comentários e cartas ao leitor/editor; e que não atendessem aos critérios de inclusão descritos.

A primeira etapa de seleção foi identificar e retirar as duplicatas. Em seguida, analisar os potenciais estudos e se preenchiam os critérios estabelecidos, passando por uma seleção inicial efetivada com leitura do título e resumo para verificar se enquadravam na temática. Após essa etapa, os estudos previamente selecionados foram lidos na íntegra, empregando os critérios de inclusão e exclusão, para análise e obtenção das informações necessárias para a construção do presente estudo. Caso houvesse discordância entre os avaliadores sobre os critérios analisados, foi realizada discussão específica sobre o artigo em questão, confrontando ideias com base nos critérios adotados para a revisão. Ao final da seleção foram incluídos 78 estudos mais recentes publicados (Tabela 1). Os estudos incluídos na revisão integrativa permitem uma avaliação crítica do tema discutido sendo possível identificar lacunas que poderão direcionar futuras pesquisas.

Tabela 1. Estudos encontrados nas buscas realizadas nas bases de dados.

\begin{tabular}{|c|c|c|}
\hline FONTES DE INFORMAÇÃO & ESTRATÉGIA DE BUSCA & RESULTADOS \\
\hline PubMed & ("printing, three dimensional"[MeSH Terms] OR ("printing"[All Fields] & 320 \\
\hline LILACS & $\begin{array}{c}\text { AND "three dimensional"[All Fields]) OR "three-dimensional } \\
\text { printing"[AllFields] OR ("3d"[All Fields] AND "printing"[All Fields]) }\end{array}$ & 176 \\
\hline SciELO & $\begin{array}{l}\text { OR "3d printing"[All Fields]) AND ("anatomy and histology"[MeSH } \\
\text { Subheading] OR ("anatomy"[All Fields] AND "histology"[All Fields]) }\end{array}$ & 2 \\
\hline Google Acadêmico & $\begin{array}{l}\text { OR "anatomy andhistology"[All Fields] OR "anatomy"[All Fields] OR } \\
\text { "anatomy"[MeSH Terms] OR "anatomies"[All Fields]) AND ("education, } \\
\text { medical"[MeSH Terms] OR ("education"[All Fields] AND "medical"[All } \\
\text { Fields]) OR "medical education"[All Fields] OR ("medical"[All Fields] } \\
\text { AND "education"[All Fields])) }\end{array}$ & 31.900 \\
\hline & Artigos selecionados incluídos na revisão: & 78 \\
\hline
\end{tabular}

Fonte: Autores (2021).

\section{Resultados e Discussão}

A tecnologia da impressão digital iniciou no final do século XX. A Impressão 3D foi concebida por um engenheiro físico da Califórnia - EUA, Charles (Chuck) W. Hull em 1984, utilizando a estereolitografia (SLA - stereolithography apparatus) patenteada por ele em 1986, que revolucionou áreas como a indústria e a engenharia, e agora na educação 
principalmente com o crescente uso dessa tecnologia na área da saúde (Volpato et al., 2017; Mumith et al., 2018; Erolin, 2019). As três tecnologias de impressão 3D mais conhecidas são a SLA, a Sinterização Seletiva a Laser ou Fusão (SLS selective laser sintering / SLM - selective laser melting) e Modelagem de Deposição Fundida (FDM - fused deposition modeling) (Silva et al., 2020). A prototipagem rápida passa por um processo sistemático necessitando de geometria espacial definida por modelos matemáticos feito por softwares do tipo CAD (computer-aided design) ou engenharia reversa, conversão em arquivo STL (surface tessellation language), transferência para o equipamento de impressão e sua manipulação, configuração dos parâmetros de impressão, remoção da peça, pós-processamento para intervenções adicionais como lixamentos adicionais e limpeza de acabamentos mais delicados (Chantarapanich et al., 2017; Corazza et al., 2020; Araujo et al., 2021).

Os primeiros tipos de materiais utilizados foram os plásticos sintéticos com menor resistência a temperatura, umidade e a intemperismos ambientais. Atualmente variados tipos de materiais podem ser utilizados na indústria 3D como o vidro, aço, cerâmicas, e até mesmo a madeira. As impressoras de "uso doméstico" ou desktop utilizam materiais como o termoplástico ácido polilático (PLA - polylactic acid) um polímero biodegradável. É de baixo custo, rígido e resistente inclusive a impactos, tem boa precisão dimensional e permite acabamentos. No entanto, apresenta baixa resistência ao calor, não é indicado para exposição a luz solar e o filamento pode ficar quebradiço com o tempo. $\mathrm{O}$ ácido láctico para sintetizar o PLA tem origem em fontes naturais renováveis que contêm amido como milho, trigo, cana-de-açúcar, beterraba ou batata (Araujo et al., 2019; Castelo Branco et al., 2021).

Já o acrilonitrila butadieno estireno (ABS - acrylonitrile butadiene styrene) é um termoplástico derivado do petróleo, com maior índice de toxicidade. Tem baixo custo, resistência a impactos, rigidez a desgaste, mas não é bom para detalhes pequenos. O uso desses materiais apresenta riscos de emissão de partículas ultrafinas (com menos de 100nm) e de compostos orgânicos voláteis perigosos. A ventilação é recomendada em caso de utilização do PLA e obrigatória se for utilizado o ABS (Barreto, 2018; Duarte et al., 2019). Os filamentos de PLA e ABS são os principais materiais utilizados em impressoras 3D, mas existem outros como o Politereftalato de Etileno Glicol (PETG) um termoplástico derivado do petróleo, um poliéster modificado com a adição de uma molécula de glicol que reduz a cristalinidade da resina final e aumenta a ductilidade e resistência ao impacto. É transparente e permite a confecção de peças resistentes e com detalhes. O Nylon também tem sido utilizado na impressão 3D, um material resistente e flexível que proporciona bom acabamento. Outra questão presente no mercado ainda é a pequena variedade de cores disponíveis desses materiais e, muitas vezes a necessidade da capacitação em design e em processos produtivos para a criação e desenvolvimento dos objetos utilizando a engenharia reversa e fotogrametria (Rodrigues et al., 2017; Jones, 2019; Araujo et al., 2021; Duarte et al., 2021).

Além do custo com os materiais que podem ser utilizados na impressão 3D outra questão que merece atenção é o custo do equipamento de impressão 3D. Há vantagens no uso dessa tecnologia como o potencial de evolução e a produção de materiais com peso reduzido, redução do uso de matéria-prima, substituição de outros processos que são energicamente ineficientes, a possibilidade de produção próxima ao consumidor final, capacidade de individualização do produto com características mais complexas, entre tantas outras características (Kim et al., 2016; Lim et al., 2016; Rodrigues et al., 2017).

Embora já muito difundida, a tecnologia da impressão 3D ainda exibe fatores limitantes para sua implementação (Silva et al., 2020). Muitas impressoras ainda apresentam custo elevado inviabilizando o uso da tecnologia. Essa realidade tende a mudar com a crescente demanda e desenvolvimento de impressoras do tipo "RepRap" que possibilidade a produção de impressoras de baixo custo (Figura 1). Esse tipo de impressora possibilita tanto a reprodução de peças, com baixo custo, como possibilita reproduzir as peças para construir uma nova impressora desse mesmo modelo (Balzani, 2017). 
Figura 1: Impressora Reprap Prusa Mendel construída por Renan Balzani e Leonardo Barreto.

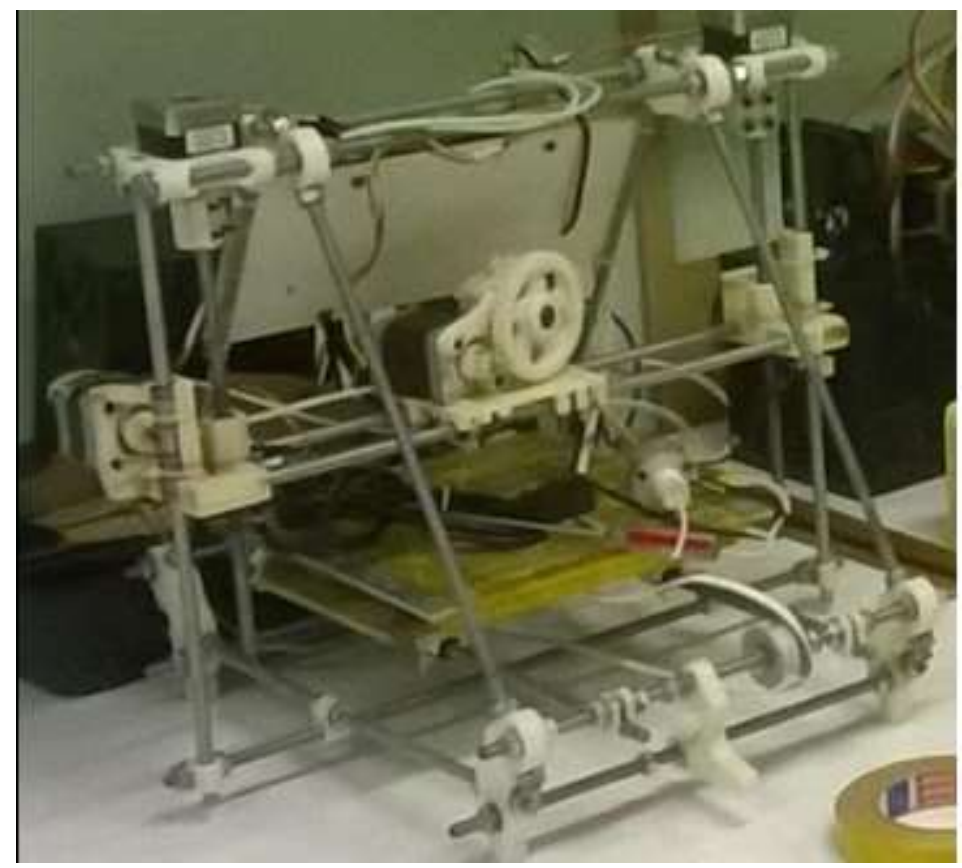

Fonte: Balzani (2017).

A tecnologia de impressão 3D avança continuamente e em 2020 foi criado o Laboratório de Inovação 3D na Universidade Evangélica de Goiás, na cidade de Anápolis. Esse laboratório possui uma impressora tridimensional do tipo Cliever CL2 Educacional (Figura 2) para produção de peças e uso pelos estudantes da universidade (UniEvangélica, 2020). Essa impressora tem tecnologia de impressão por Filamento Fundido (FDM/FFF) e mesa de impressão com superfície de vidro aquecida, e pode trabalhar com ABS, PETG e PLA. Peças anatômicas já foram desenvolvidas utilizando imagens de exames reais, com base no InVesalius ${ }^{\circledR}$, um software livre do Ministério da Ciência, Tecnologia e Inovações para reconstrução de imagens provindas de equipamentos de tomografia computadorizada ou ressonância magnética, e foram produzidas para estudos e pesquisas de iniciação científica, realizadas pelos alunos do curso de medicina. O nome InVesalius é uma homenagem ao médico belga Andreas Vesalius, considerado o "pai da anatomia moderna". 
Figura 2: Impressora do tipo Cliever CL2 Educacional.

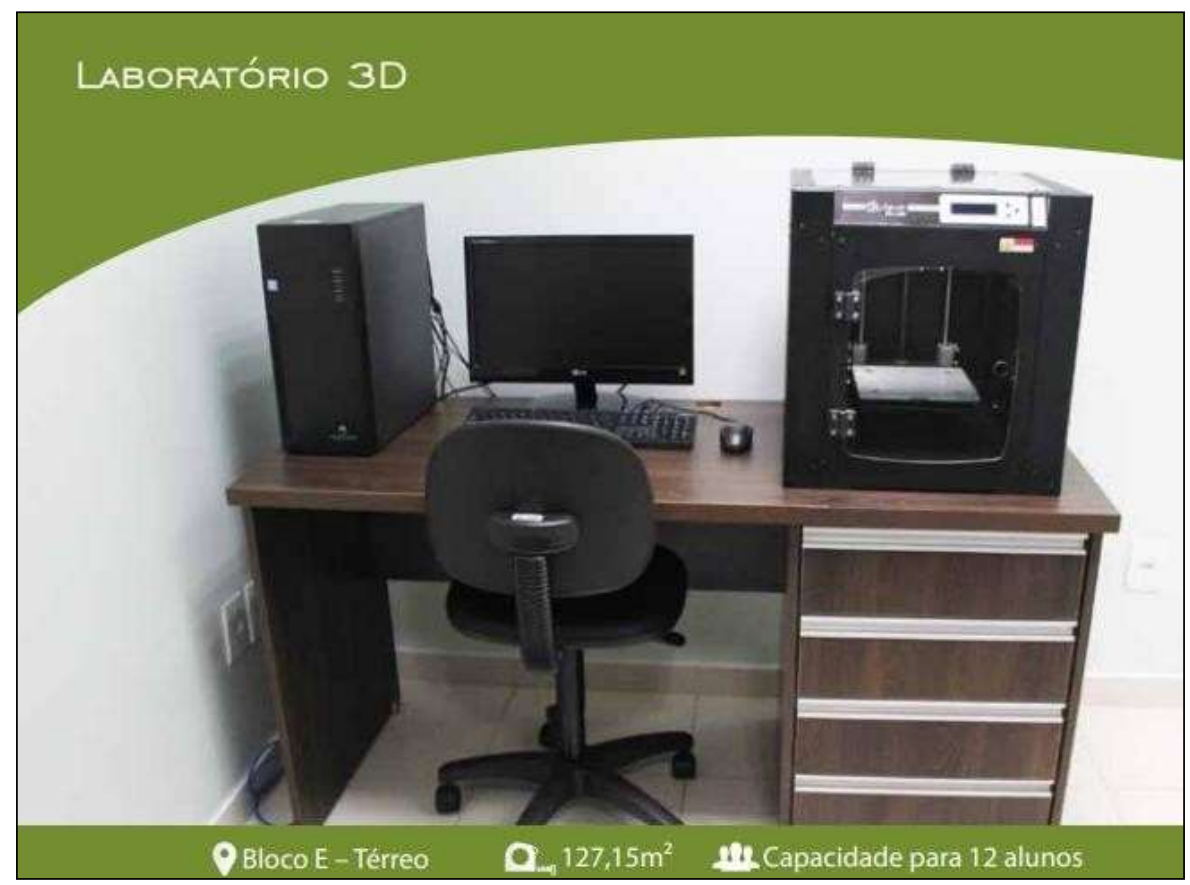

Fonte: UniEvangélica (2020).

A aplicabilidade da impressão 3D foca no desenvolvimento de novas metodologias ativas de aprendizagem, seja em anatomia ou outras disciplinas que possam utilizar os modelos criados com a modelagem 3D (Keenan \& Ben Awadh, 2019; Louredo et al., 2019). Essas possibilidades mostram a evolução da tecnologia. Hoje já existem impressoras 4D, que surgiram a partir da 3D, mas o material produzido "muda" sua forma posteriormente. Além dos aspectos dimensionais, volumétricos e de produção, adiciona-se a função tempo fazendo com que os materiais que antes eram rígidos, sólidos, sem alterações ao longo do tempo, passem a ter sua forma alterada dentro da linha do tempo (Wang et al., 2021b). Estímulos como pressão, temperatura, umidade, luminosamente fazem com que o material impresso mude de forma, função, cor, e até de função (Choi et al., 2015; Shakibania et al., 2021).

\subsection{Modelos tridimensionais no processo de ensino e aprendizagem}

A inovação dos recursos tecnológicos gera cada vez mais informações e conhecimentos científicos. A complexidade e os detalhes de cada desdobramento de uma determinada área admite o desenvolvimento de novos métodos de ensino, principalmente aqueles promovidos por novas metodologias. A anatomia é uma área estudada por todos os cursos da área da saúde, mais aprofundada por uns, ou menos por outros, mas todos precisam saber sobre particularidades dos sistemas corporais. Contudo, para alguns estudantes da área da saúde estudar anatomia é mais difícil devido ao escasso acesso a peças anatômicas reais, a dificuldade de correlacionar dados clínicos com os conhecimentos básicos, além da necessidade da estimular o trabalho em equipe e raciocínio clínico durante as aulas práticas de anatomia humana (Keenan \& Ben Awadh, 2019).

Nesse sentido, Wen (2016) desenvolveu o Projeto Homem Virtual ou Ser Humano Virtual, iniciado no Laboratório de Inovação e Mídias Interativas da Disciplina de Telemedicina do Departamento de Patologia da Faculdade de Medicina da USP, com o intuito de adequar os critérios de aprendizagem de uma forma ampla, associando anatomia, biomecânica, 
fisiologia entre outras áreas, por meio da computação gráfica 3D e impressão digital, reduzindo o tempo de ensino dos conteúdos teóricos densos e a ampliação da prática de outras habilidades como raciocínio, análise clínica e contextualização prática.

A metodologia de ensino utilizando impressão 3D foi aplicada por Barreto (2018) em estudantes de medicina da Escola Baiana de Medicina e Saúde Pública, comparando estudantes que utilizaram o método de impressão 3D com outro grupo que utilizou o método tradicional de estudo. Os resultados indicaram que os modelos 3D foram um recurso pedagógico bem aceito pelos estudantes e professores, visto que a nova metodologia proporcionou uma melhor compreensão da forma e das relações espaciais, além do auxílio no entendimento de variações anatômicas.

Cui et al., (2017) utilizaram modelos vasculares 3D no ensino de anatomia da circulação de cabeça e pescoço, com estudantes do primeiro ano de medicina do Centro Médico da Universidade de Mississippi. Foram aplicadas avaliações pré e pós sessão de aprendizagem, como também de habilidades espaciais. Os resultados mostraram que todos os alunos que foram expostos aos modelos vasculares 3D aumentaram sua capacidade de identificar corretamente a anatomia vascular de cabeça e pescoço. E o mais importante, os alunos com baixa capacidade espacial melhoraram o conhecimento pós sessão com modelo 3D, indicando que o uso de modelos é um instrumento valioso para esses alunos com baixa capacidade de compreensão espacial. Outro grupo publicou no $9^{\circ}$ Information Design International Conference um modelo anatômico da $11^{\text {a }}$ vértebra torácica (T-11), por meio do escaneamento com tecnologia bluelight, um sistema que usa pontos de referência para a digitalização do objeto tridimensional (Romeiro et al., 2019). Foram realizadas tanto com a técnica de impressão com resina quanto com filamento (Figura 3) mostrando como essa tecnologia democratiza o acesso ao conhecimento anatômico nos diversos ramos da ciência mesmo com questões que necessitam de melhoria ao longo do tempo.

Figura 3: Modelo impresso utilizando filamento (esquerda) e impresso com resina (direita).

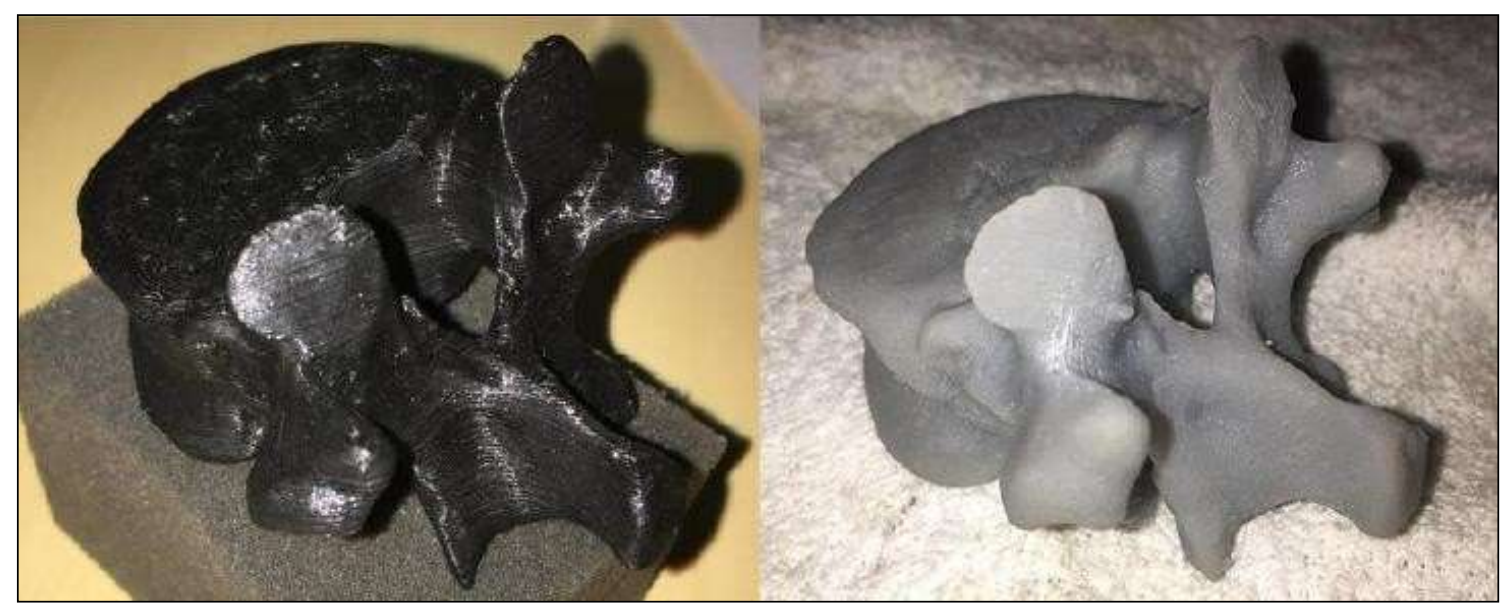

Fonte: Romeiro et al. (2019).

A eficácia do uso de modelos tridimensionais para ensinar anatomia é um tópico de muita discussão na pesquisa em educação médica (Erolin, 2019). Para além das aplicações na área médica, a tecnologia manufatura aditiva para estudo de anatomia pode ser aplicada em outras áreas (Mumith et al., 2018). A reconstrução tridimensional e a impressão de biomodelos de um crânio canino foram feitos a partir de exames de TC, exportadas e modeladas com a remoção de defeitos, artefatos e partes indesejadas no Hospital Veterinário da Faculdade de Medicina Veterinária e Zootecnia da USP. Esse desafio de processamento depende de um estudo um pouco mais aprofundado em modelagem, produzindo modelos com $60 \%$ e $100 \%$ de 
escala com qualidade e destalhes muito similares ao exemplo real (Bertti et al., 2020).

Evoluindo nos critérios de aprendizagem, o próximo passo após o estudo de anatomia e fisiologia dentro do aspecto da normalidade, é a visualização, entendimento e aplicabilidade desses conhecimentos na resolução dos casos clínicos (Ye et al., 2020). Nesse sentido, a impressão 3D pode corroborar de forma muito positiva (Su et al., 2018; Neumann et al., 2019). Com o uso de ecocardiograma transesofágico, biomodelos foram produzidos com características normais, isquêmicas e degeneradas de uma válvula bicúspide (Figura 4). Esses protótipos foram gerados com grande acurácia de similaridade anatômica, mesmo com limitações como a replicação da dinâmica do tecido e sua fisiologia (Banerjee et al., 2020). O desenvolvimento de materiais coloridos e flexíveis para impressão em 3D tornou possível a aplicação do estudo de patologias específicas, além do planejamento cirúrgico de tecidos moles de maneira significativa (Mahmood et al., 2015; Valverde, 2017; Ganguli et al., 2018; Lugassy et al., 2020; Leary et al., 2021).

Figura 4: A esquerda válvula bicúspide normal impressa, no centro válvula bicúspide isquêmica, e a direita válvula bicúspide com degeneração mixomatosa (perda da elasticidade).

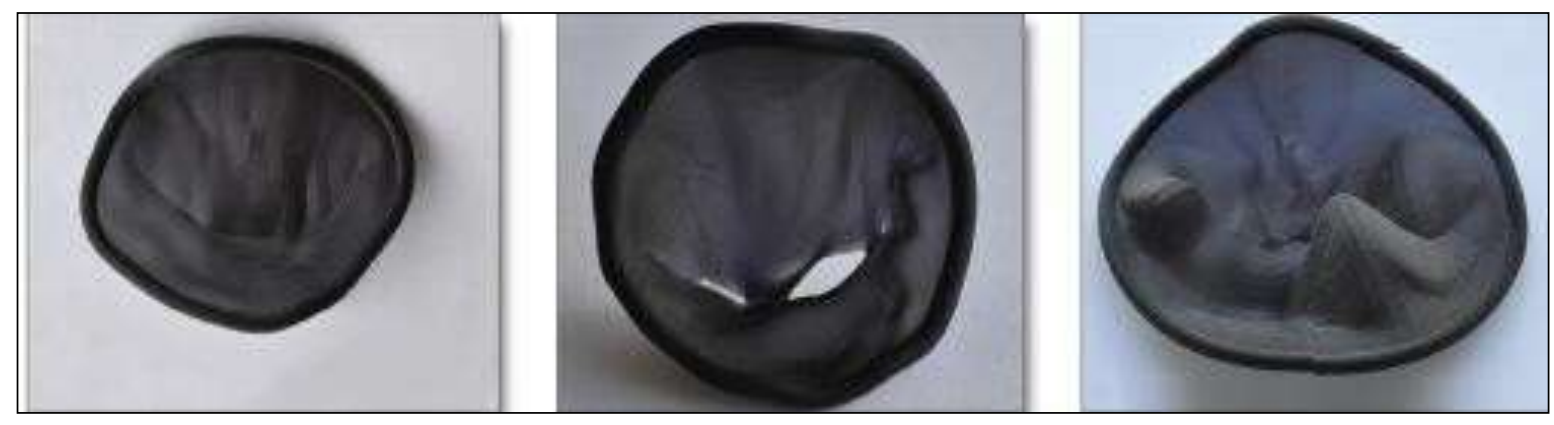

Fonte: Mahmood et al. (2015).

$\mathrm{Na}$ medicina cardiovascular o interesse da aplicabilidade da tecnologia tridimensional continua aumentando (Valverde, 2017; Su et al., 2018; Noor et al., 2019). Com uso de TC e ecocardiograma cardíaco, biomodelos de válvulas atrioventriculares foram feitos a partir de quatro técnicas e materiais: modelagem de deposição fundida (FDM), na qual as camadas são feitas pela extrusão de material sólido; estereolitografia (SLA) as camadas são feitas com resina líquida por fotopolimerização; sinterização seletiva a laser (SLS) em que um laser funde as camadas com um pó de polímeros termoplásticos granulares; e o uso de PolyJet que usa resinas líquidas de fotopolímeros que solidificam na presença de luz ultravioleta, mas com capacidade de produzir materiais flexíveis. Comparando essas técnicas observou-se precisão submilimétrica entre os biomodelos e a proporção real do órgão e de problemas cardíacos associados como as calcificações, e 55\% dos profissionais de saúde avaliados aprovaram os modelos para estudos anatômicos e clínicos (Birbara et al., 2019).

O benefício do uso da tecnologia 3D para estudo e planejamento cirúrgico começou com a idealização de estruturas sólidas, rígidas como nas cirurgias craniofacial, maxilofacial e até de coluna vertebral, chegando a ser utilizada em cirurgias mais complexas como as cardiovasculares, do abdome e neurocirurgias (Bizzotto et al., 2015; Kim et al., 2016; Elomaa \& Yang, 2017; Bartikian et al., 2019; Miljanovic et al., 2020). A possibilidade de replicação de órgãos com o intuito de estudo, definição de condutas cirúrgicas mostra-se cada vez mais comum.

Cirurgias robóticas já são realidade em muitos países (Garcia et al., 2018; Uwechue et al., 2018; Ghazi \& Teplitz, 2020). Um modelo de aneurisma de aorta foi criado com resinas translucidas, por meio de imagens de TC, associada a focos de calcificação e a presença de trombo mural, cada parte anatômica possuía cor própria possibilitando o estudo de estruturas 
intraparenquimatosas de forma mais realista indicando melhor planejamento de atuação (Jones et al., 2016; Taher et al., 2017; Chen et al., 2020). Materiais transparentes, coloridos e flexíveis para impressão em 3D permitem um planejamento cirúrgico de tecidos moles de maneira mais significativa, como as características normais, isquêmicas e degeneradas de uma válvula, protótipos com grande acurácia de similaridade anatômica, mesmo com limitações como a replicação da dinâmica do tecido e sua fisiologia (Mahmood et al., 2015; Mao et al., 2015; Ballard et al., 2018; Sheu et al., 2019; Lugassy et al., 2020).

Artérias femorais para o treinamento via ultrassonografia (USG) para acesso central foi o objetivo de um estudo que utilizou imagens de tomografia computadorizada para construir os modelos (Figura 5). Os estudantes foram randomizados entre um grupo que utilizou no treinamento a simulação com o modelo e outro sem o modelo 3D. Os resultados mostraram aumento da satisfação e da confiança com o uso do modelo nos testes (Sheu et al., 2019). O planejamento cirúrgico em artroplastias totais mostrou melhoria na intervenção e na redução de complicações como infecções e tromboses. Compreender as proporções reais da anatomia melhora os acessos cirúrgicos como o aumento da precisão da ressecção de tumores e o planejamento de fixação ortopédicas (Mao et al., 2015; Ballard et al., 2018).

Figura 5: Modelo 3D da anatomia óssea associada a tecido mole dos vasos e ao gel visível ao ultrassom.

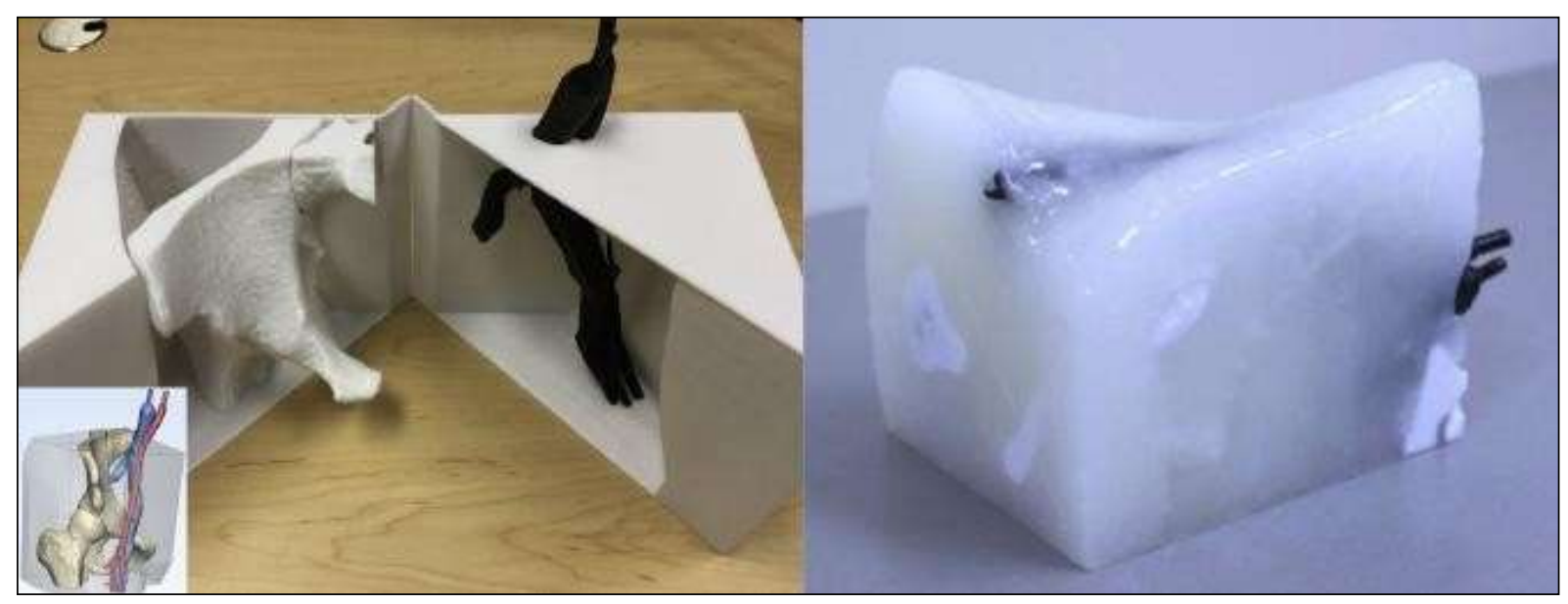

Fonte: Sheu et al. (2019).

A aplicabilidade da impressão 3D apresenta níveis de produção incontáveis desde o planejamento cirúrgico, peças com similaridade ao real, quanto ao desenvolvimento de técnicas e treinamento para cirurgias complexas com materiais muito próximos ao in vivo (Ganguli et al., 2018; Leary et al., 2021). No campo da urologia, a decisão e o treinamento cirúrgico são paciente-dependentes (Ghazi \& Teplitz, 2020). Com a reprodução de biomodelos impressos do rim de um paciente, Smith e Dasgupta (2020), concluíram que houve melhor identificação do problema estrutural a ser solucionado, um melhor entendimento da capilaridade vascular reduzindo o tempo cirúrgico e a duração da isquemia intraoperatória, e melhor performance cirúrgica, como também redução do uso de fluoroscopia e erros na punção percutânea com treinamentos realizados em biomodelos da nefrolitotripsia percutânea.

O planejamento cirúrgico com o uso dos modelos tridimensionais segue como um grande avanço da medicina (Garcia et al., 2018; Wilk et al., 2020). Na preparação pré-operatória é possível determinar a melhor escolha do uso de próteses no tratamento de aneurismas. Com a comparação de modelos tridimensionais a escolha e a alteração do enxerto endógeno fenestrado foi possível em tratamento de aneurismas da aorta abdominal com zona de aterrissagem infrarrenal insuficiente, de forma personalizada sendo que a cada cinco endopróteses testadas em comparação com o modelo 3D, uma apresentou 
alterações antes da aplicação (Taher et al., 2017).

Já foram descritas diversas técnicas em que a manufatura aditiva propõe a impressão de tecidos vivos. Principalmente pela estruturação de imagens em 3D, por meio de arquivos CAD que, com impressoras do tipo jato de tinta, é possível imprimir materiais biológicos contendo células vivas. Exemplos dessas técnicas são: deposição de camadas com uso de bioink ou "biotintas" (uma formulação de células), extrusão por seringa, e até a soma das outras técnicas utilizadas na produção atual de enxertos convencionais em combinação com a técnica da impressão digital (Elomaa \& Yang, 2017; Liu \& Wang, 2020).

A construção de estruturas viáveis e órgãos por meio da tecnologia de impressão já é uma realidade. A construção tecidual de vasos dentro da engenharia de tecidos é uma opção. A concepção de vasos artificiais por meio da tecnologia 3D com o uso dos "andaimes" ou "scaffords" possibilitam direcionar a colonização e a proliferação celular (Itoh et al., 2015). Foi possível com a MA, moldado pela técnica de empilhamento, a construção de materiais mais complexos que antes não eram viáveis, mesmo com algumas limitações como o dimensionamento tecidual (Hann et al., 2019). Usando a tecnologia da engenharia de tecidos, Luo et al. (2020) recriaram vasos sanguíneos com a formação de células musculares lisas de vasos pela transformação de células-tronco pluripotentes humanas. Tais células foram associados a uma base, uma estrutura de sustentação que mantém a tridimensionalidade do tecido, os "bioscaffolds" de ácido poliglicólico biodegradável (PGA - poly glycolic acid). Esses vasos seguiram os critérios descritos por Niu et al. (2014) que adotaram as características mecânicas, celulares e imunológicas similares aos vasos originais do hospedeiro ou similares aos enxertos autólogos, já usados no dia a dia da cirurgia cardiovascular.

Wang et al. (2021a) descreveram que a construção de tecidos, principalmente vasos, com a técnica de andaimes, faz uso da engenharia reversa com a transformação de exames de imagem como micro-tomografia computadorizada e ressonância magnética em modelos tridimensionais. Esse modelo reproduz de forma fiel as estruturas in vivo com apresentação precisa das formas anatômicas individuais. Por outro lado, o uso do design paramétrico, método com o uso de softwares e algoritmos prédeterminados na manufatura auxiliada por computador (CAM - computer aided manufacturing), também é uma forma para a construção de um modelo tridimensional viável, principalmente quando associado com inteligência artificial (Mumith et al., 2018; Kriegman et al., 2020; Wang et al., 2021b).

É possível ainda produzir estruturas teciduais sem o auxílio desses andaimes. O cultivo de cardiomiócitos derivados de células-tronco pluripotentes induzidas, células endoteliais e fibroblastos foi possível pela formação de esferoides de células passíveis de serem colocados em agulhas de impressoras Bio-3D que possibilitou a recriação direta tridimensional de modelos de válvulas cardíacas viáveis funcionalmente (Arai et al., 2018). Usando essa mesma tecnologia, foi possível, com o uso de células endoteliais da veia umbilical humana, células do músculo liso da aorta humana e fibroblastos dérmicos humanos normais; a produção de um modelo aórtico abdominal. Esse modelo, aplicado em ratos, mostrou-se viável principalmente pela sua capacidade própria de remodelação e endotelização ao tecido implantado (Itoh et al., 2015; Liu \& Wang, 2020).

Muitos desafios ainda precisam ser suplantados. A reprodução de modelos 3D digitais por meio do uso da angiografia coronária invasiva para a aplicação de cateterismos ainda não apresenta precisão devido a variação cinética do aparelho respiratório e cardiovascular no momento do exame e principalmente pela sobreposição de diversos vasos. Com isso, a introdução de um algoritmo de pontos de nuvem possibilitou reduzir os erros de projeções resultando em grande precisão nos testes (Banerjee et al., 2020).

O uso dessa técnica de impressão 3D na preparação cirúrgica, na popularização do ensino anatômico, na produção de tecidos e órgãos individualizados com características bioquímicas e imunológicas do próprio hospedeiro com a resolução de 
patologias antes de difícil tratamento está cada vez mais próximo. A aplicação do produto tridimensional no contexto do processo ensino-aprendizado ainda se apresenta como um desafio. A presença mesmo que sutil da impressão no cenário do ensino médico quanto de outras áreas precisa melhorar. Entretanto, essa mudança de ambiente é bem promissora. Nesse sentido é perceptível que o crescimento científico será exponencial e que, dessa forma, estaremos cada vez mais próximo de uma nova realidade.

\section{Considerações Finais}

Um modelo tridimensional com suas características volumétricas são fundamentais para a percepção da realidade espacial. Dentro do processo de ensino e aprendizagem nos campos da área da saúde a anatomia humana tanto fascina como assusta, mas é um dos primeiros passos que o estudante dá para seguir nessa área. A partir disso, a cada degrau alcançado novos desafios são impostos e vão desde a compreensão do que é a normalidade de um sistema funcional, a composição dos órgãos, tecidos e células até a compreensão dos distúrbios envolvidos, o treinamento prático necessário e sua correlação com a realidade clínica e cirúrgica quando necessário.

Nesse sentido, as limitações dos métodos tradicionais que envolvem estudos com visualizações bidimensionais, ilustrações ou a escassez de peças anatômicas reais como os cadáveres, e muitas vezes até mesmo o envolvimento direto com o paciente sem treinamento consolidado, despontam a necessidade de alternativas para a aquisição de mais conhecimentos. Os métodos de inovação, como a aplicação da impressão digital, permitiram atingir um novo patamar. Correlações clínicas a partirda realidade de um caso médico, produção de modelos com precisão muito próxima ao real, melhoria de habilidades visuais, espaciais, raciocínio, planejamento são a ponta do iceberg no que tange a aplicabilidade dessa nova apresentação metodológica.

Mesmo com esse grande avanço, os desafios permanecem. $\mathrm{O}$ acesso mesmo que facilitado nas últimas décadas, ainda não atinge de forma satisfatória a população. O custo do equipamento e do material de consumo/mão de obra, mesmo com redução, ainda se tornam um empecilho. A necessidade de um treinamento para a produção dos biomodelos ainda limita a confecção de ideias. Todavia, com o desenvolvimento tecnológico, para além da produção de modelos que variam ao longo do tempo, como a tecnologia $4 \mathrm{D}$, e da capacidade de produzir materiais biológicos para auxiliar o campo da medicina; a utilização da tecnologia 3D tende a se democratizar. Nesse sentido, o presente estudo aponta a relevância do tema, a impressão tridimensional veio para ficar e está sendo cada vez mais aplicada.

\section{Referências}

Allen, L. K., Bhattacharyya, S., \& Wilson, T. D. (2015). Development of an interactive anatomical three-dimensional eye model. Anatomical sciences education, 8(3), 275-282. https://doi.org/10.1002/ase.1487

Arai, K., Murata, D., Verissimo, A. R., Mukae, Y., Itoh, M., Nakamura, A., Morita, S., \& Nakayama, K. (2018). Fabrication of scaffold-free tubular cardiac constructs using a Bio-3D printer. PloS one, 13(12), e0209162. https://doi.org/10.1371/journal.pone.0209162

Araujo, M. C. E., Duarte, M. M. S., Louredo, L. M., Louredo, J. M., \& Arruda, J. T. (2021). Contribuições da engenharia reversa e produção de modelos 3D para o ensino médico. Research, Society and Development, 10(11), e385101119692. https://doi.org/10.33448/rsd-v10i11.19692

Araujo, M. C. E., Louredo, L. M., Duarte, M. M. S., Moreira, S. M., Sugita, D. M., \& Arruda, J. T. (2019). Uso da engenharia reversa e tecnologia 3D para produção de biomodelos a partir de exames de imagem reais. ANAIS I CAMEG., RESU - Revista Educação em Saúde, 7, suplemento 3.

Awadh, A. B., Clark, J., Clowry, G., \& Keenan, I. D. (2020). Multimodal Three-Dimensional Visualization Enhances Novice Learner Interpretation of Basic Cross-Sectional Anatomy. Anatomical sciences education, 10.1002/ase.2045. Advance online publication. https://doi.org/10.1002/ase.2045.

Balestrini, C., \& Campo-Celaya, T. (2016). With the advent of domestic 3-dimensional (3D) printers and their associated reduced cost, is it now time for every medical school to have their own 3D printer? Medical Teacher, 38(3), 312-313. https://doi.org/10.3109/0142159X.2015.1060305. 
Ballard, D. H., Trace, A. P., Ali, S., Hodgdon, T., Zygmont, M. E., DeBenedectis, C. M., Smith, S. E., Richardson, M. L., Patel, M. J., Decker, S. J., \& Lenchik, L. (2018). Clinical Applications of 3D Printing: Primer for Radiologists. Academic radiology, 25(1), 52-65. https://doi.org/10.1016/j.acra.2017.08.004

Balzani, R. N. (2017). A produção de impressoras tridimensionais de baixo custo para estudantes de arquitetura. Dissertação de mestrado. Programa de PósGraduação da Faculdade de Arquitetura e Urbanismo da Universidade de Brasília. Brasília, 100 p.

Banerjee, A., Galassi, F., Zacur, E., De Maria, G. L., Choudhury, R. P., \& Grau, V. (2020). Point-Cloud Method for Automated 3D Coronary Tree Reconstruction From Multiple Non-Simultaneous Angiographic Projections. IEEE transactions on medical imaging, 39(4), 1278-1290. https://doi.org/10.1109/TMI.2019.2944092

Barreto, T. F. (2018). Uso de peças anatômicas em 3d como estratégia para o ensino da anatomia em curso médico. Dissertação de mestrado. Escola Baiana de Medicina e Saúde Pública, Salvador, BA, Brasil, 85 p.

Bartikian, M., Ferreira, A. Gonçalves-Ferreira, A. \& Neto, L. L. (2019). 3D printing anatomical models of head bones. Surgical and Radiologic Anatomy, 41(10), 1205-1209. https://doi.org/10.1007/s00276-018-2148-4.

Bertti, J. V. P., Silveira, E. E., \& Neto, A. C. A. (2018). Reconstrução e impressão 3D do neurocrânio de cão com o uso de tomografia computadorizada como ferramenta para auxiliar no ensino da anatomia veterinária. Arquivo Brasileiro de Medicina Veterinária e Zootecnia, 75(5), p. 1653-1658. http://dx.doi.org/10.1590/1678-4162-11209

Birbara, N. S., Otton, J. M., \& Pather, N. (2019). 3D Modelling and Printing Technology to Produce Patient-Specific 3D Models. Heart, lung \& circulation, 28(2), 302-313. https://doi.org/10.1016/j.hlc.2017.10.017

Bizzotto, N., Sandri, A., Regis, D., Romani, D., Tami, I., \& Magnan, B. (2015). Three-Dimensional Printing of Bone Fractures: A New Tangible Realistic Way for Preoperative Planning and Education. Surgical innovation, 22(5), 548-551. https://doi.org/10.1177/1553350614547773

Castelo Branco, R. R., Martins, K. Y. N., Filgueira, A. K. L., Valadares, E. J. O., Galdino, K. E., Morais, M. E.., Ramos, M. G. O., Martins, N. N., Martins, K. Y. N., \& Rodrigues, J. K. G. (2021). Caracterização da performance do material Poliácido Lático (PLA) manufaturado pela tecnologia de Modelagem de Fusão e Deposição (FDM). Research, Society and Development, 10(8), e44210817348. https://doi.org/10.33448/rsd-v10i8.17348

Chantarapanich, N., Rojanasthien, S., Chernchujit, B., Mahaisavariya, B., Karunratanakul, K., Chalermkarnnon, P., Glunrawd, C., \& Sitthiseripratip, K. (2017). 3D CAD/reverse engineering technique for assessment of Thai morphology: Proximal femur and acetabulum. Journal of Orthopaedic Science, 22(1), 703-709. https://doi.org/10.1016/j.jos.2017.02.003.

Chen, Y., Bian, L., Zhou, H., Wu, D., Xu, J., Gu, C., Fan, X., Liu, Z., Zou, J., Xia, J., \& Xu, Z. (2020a). Usefulness of three-dimensional printing of superior mesenteric vessels in right hemicolon cancer surgery. Scientific reports, 10(1), 11660. https://doi.org/10.1038/s41598-020-68578-y

Choi, J. Kwon, O. Jo, W. Lee, H. J., \& Moon, M. (2015). 4D Printing Technology: A Review. 3D Printing and Additive Manufacturing. 2(4), 159-167. https://doi.org/10.1089/3dp.2015.0039

Corazza, P. F. L., Baeder, F. M., Silva, D. F., Albuquerque, A. C. L., Silva, J. V. L., Junqueira, J. L. C., \& Panzarella, F. K. (2020). Avaliação da precisão de diferentes protocolos de aquisição de TCFC usados em modelos de prototipagem rápida. Research, Society and Development, 9(11), e2649119842. https://doi.org/10.33448/rsd-v9i11.9842

Cordeiro, R. G. \& Menezes, R. F. (2019). A Falta de Cadáveres para Ensino e Pesquisa. Revista Brasileira de Educação Médica. 43(1), 588-597. https://doi.org/10.1590/1981-5271v43suplemento1-20190217

Cramer, J., Quigley, E., Hutchins, T., \& Shah, L. (2017). Educational Material for 3D Visualization of Spine Procedures: Methods for Creation and Dissemination. Journal of digital imaging, 30(3), 296-300. https://doi.org/10.1007/s10278-017-9950-0

Cui, D., Wilson, T. D., Rockhold, R. W., Lehman, M. N., \& Lynch, J. C. (2017). Evaluation of the effectiveness of 3D vascular stereoscopic models in anatomy instruction for first year medical students. Anatomical sciences education, 10(1), 34-45. https://doi.org/10.1002/ase.1626

Duarte, M. M. S., Araujo, M. C. E., Louredo, L. M., Louredo, J. M., \& Arruda, J. T. (2021). Aplicabilidades da técnica de fotogrametria no ensino de Anatomia Humana. Research, Society and Development, 10(11), e51101119328. https://doi.org/10.33448/rsd-v10i11.19328

Duarte, M. M. S., Araújo, M. C. E., Louredo, L. M., Moreira, S. M., Sugita, D. M., \& Arruda, J. T. (2019). Fotogrametria e impressão 3D aplicada ao ensino de anatomia. ANAIS I CAMEG., RESU - Revista Educação em Saúde, 7, suplemento 3.

Elomaa, L., \& Yang, Y. P. (2017). Additive Manufacturing of Vascular Grafts and Vascularized Tissue Constructs. Tissue engineering. Part B, Reviews, 23(5), 436-450. https://doi.org/10.1089/ten.TEB.2016.0348

Erolin C. (2019). Interactive 3D Digital Models for Anatomy and Medical Education. Advances in experimental medicine and biology, 1138, 1-16. https://doi.org/10.1007/978-3-030-14227-8_1

Ganguli, A., Pagan-Diaz, G. J., Grant, L., Cvetkovic, C., Bramlet, M., Vozenilek, J., Kesavadas, T., \& Bashir, R. (2018). 3D printing for preoperative planning and surgical training: a review. Biomedical microdevices, 20(3), 65. https://doi.org/10.1007/s10544-018-0301-9.

Garcia, J., Yang, Z., Mongrain, R., Leask, R. L., \& Lachapelle, K. (2018). 3D printing materials and their use in medical education: a review of current technology and trends for the future. BMJ Simulation \& Technology Enhanced Learning, 4(1), 24-40. https://doi.org/10.1136/bmjstel-2017-000234. 
Ghazi, A. E., \& Teplitz, B. A. (2020). Role of 3D printing in surgical education for robotic urology procedures. Translational andrology and urology, 9(2), 931-941. https://doi.org/10.21037/tau.2020.01.03

Guimarães, B., Dourado, L., Tsisar, S., Diniz, J. M., Madeira, M. D., \& Ferreira, M. A. (2017). Rethinking Anatomy: How to Overcome Challenges of Medical Education's Evolution. Acta medica portuguesa, 30(2), 134-140. https://doi.org/10.20344/amp.8404

Hann, S. Y., Cui, H., Esworthy, T., Miao, S., Zhou, X., Lee, S. J., Fisher, J. P., \& Zhang, L. G. (2019). Recent advances in 3D printing: vascular network for tissue and organ regeneration. Translational research: the journal of laboratory and clinical medicine, 211, 46-63. https://doi.org/10.1016/j.trsl.2019.04.002

Hecht-López, P., \& Larrazábal-Miranda, A. (2018). Uso de Nuevos Recursos Tecnológicos en la Docencia de un Curso de Anatomía con Orientación Clínica para Estudiantes de Medicina. International Journal of Morphology, 36(3), 821-828. https://dx.doi.org/10.4067/S0717-95022018000300821.

Hermsen, J. L., Roldan-Alzate, A., \& Anagnostopoulos, P. V. (2020). Three-dimensional printing in congenital heart disease. Journal of thoracic disease, 12(3), 1194-1203. https://doi.org/10.21037/jtd.2019.10.38.

Itoh, M., Nakayama, K., Noguchi, R., Kamohara, K., Furukawa, K., Uchihashi, K., Toda, S., Oyama, J., Node, K., \& Morita, S. (2015). Scaffold-Free Tubular Tissues Created by a Bio-3D Printer Undergo Remodeling and Endothelialization when Implanted in Rat Aortae. PloS one, 10(9), e0136681. https://doi.org/10.1371/journal.pone.0136681

Jones D. G. (2019). Three-dimensional Printing in Anatomy Education: Assessing Potential Ethical Dimensions. Anatomical sciences education, 12(4), 435443. https://doi.org/10.1002/ase.1851

Jones, D. B., Sung, R., Weinberg, C., Korelitz, T., \& Andrews, R. (2016). Three-Dimensional Modeling May Improve Surgical Education and Clinical Practice. Surgical innovation, 23(2), 189-195. https://doi.org/10.1177/1553350615607641

Keenan, I. D., \& Ben Awadh, A. (2019). Integrating 3D Visualisation Technologies in Undergraduate Anatomy Education. Advances in experimental medicine and biology, 1120, 39-53. https://doi.org/10.1007/978-3-030-06070-1_4

Kim, G. B., Lee, S., Kim, H., Yang, D. H., Kim, Y. H., Kyung, Y. S., Kim, C. S., Choi, S. H., Kim, B. J., Ha, H., Kwon, S. U., \& Kim, N. (2016). ThreeDimensional Printing: Basic Principles and Applications in Medicine and Radiology. Korean journal of radiology, 17(2), 182-197. https://doi.org/10.3348/kjr.2016.17.2.182

Koche, J. C. (2011). Fundamentos de metodologia científica. Petrópolis: Vozes

Kriegman, S., Blackiston, D., Levin, M., \& Bongard, J. (2020). A scalable pipeline for designing reconfigurable organisms. Proceedings of the National Academy of Sciences of the United States of America, 117(4), 1853-1859. DOI: 10.1073/pnas.1910837117

Krishnasamy, S., Mokhtar, R., Singh, R., Sivallingam, S., Aziz, Y., \& Mathaneswaran, V. (2021). 3D Rapid Prototyping Heart Model Validation for Teaching and Training - A Pilot Project in a Teaching Institution. Brazilian journal of cardiovascular surgery, 10.21470/1678-9741-2020-0433. Advance online publication. https://doi.org/10.21470/1678-9741-2020-0433

Leary, O. P., Crozier, J., Liu, D. D., Niu, T., Pertsch, N. J., Camara-Quintana, J. Q., Svokos, K. A., Syed, S., Telfeian, A. E., Oyelese, A. A., Woo, A. S., Gokaslan, Z. L., \& Fridley, J. S. (2021). Three-Dimensional Printed Anatomic Modeling for Surgical Planning and Real-Time Operative Guidance in Complex Primary Spinal Column Tumors: Single-Center Experience and Case Series. World neurosurgery, 145, e116-e126. https://doi.org/10.1016/j.wneu.2020.09.145.

Lim, K. H., Loo, Z. Y., Goldie, S. J., Adams, J. W., \& McMenamin, P. G. (2016). Use of 3D printed models in medical education: A randomized control trial comparing 3D prints versus cadaveric materials for learning external cardiac anatomy. Anatomical sciences education, 9(3), 213-221. https://doi.org/10.1002/ase.1573

Liu, F., \& Wang, X. (2020). Synthetic Polymers for Organ 3D Printing. Polymers, 12(8), 1765. https://doi.org/10.3390/polym12081765

Louredo, L. M, Duarte, M. M. S., Araújo, M. C. E., Moreira, S. M., Sugita, D. M., \& Arruda, J. T. (2019). Aplicabilidade de biomodelos tridimensionais produzidos com impressora 3d para estudos de anatomia. RESU - Revista Educação em Saúde: V7, suplemento 3. Recuperado de: http://periodicos.unievangelica.edu.br/index.php/educacaoemsaude/article/view/4187/3102

Lozano, M. T. U., Haro, F. B., Diaz, C. M., Manzoor, S., Ugidos, G. F., \& Mendez, J. A. J. (2017). 3D Digitization and Prototyping of the Skull for Practical Use in the Teaching of Human Anatomy. Journal of Medical Systems, 41(83), 1-5. https://doi.org/10.1007/s10916-017-0728-1.

Lugassy, D., Levanon, Y., Rosen, G., Livne, S., Fridenberg, N., Pilo, R., \& Brosh, T. (2020). Does Augmented Visual Feedback from Novel, Multicolored, Three-Dimensional-Printed Teeth Affect Dental Students' Acquisition of Manual Skills? Anatomical sciences education, 10.1002/ase.2014. Advance online publication. ttps://doi.org/10.1002/ase.2014.

Luo, J., Qin, L., Zhao, L., Gui, L., Ellis, M. W., Huang, Y., Kural, M. H., Clark, J. A., Ono, S., Wang, J., Yuan, Y., Zhang, S. M., Cong, X., Li, G., Riaz, M., Lopez, C., Hotta, A., Campbell, S., Tellides, G., Dardik, A., ... Qyang, Y. (2020). Tissue-Engineered Vascular Grafts with Advanced Mechanical Strength from Human iPSCs. Cell stem cell, 26(2), 251-261.e8. https://doi.org/10.1016/j.stem.2019.12.012

Mahmood, F., Owais, K., Taylor, C., Montealegre-Gallegos, M., Manning, W., Matyal, R., \& Khabbaz, K. R. (2015). Three-dimensional printing of mitral valve using echocardiographic data. JACC. Cardiovascular imaging, 8(2), 227-229. https://doi.org/10.1016/j.jcmg.2014.06.020

Mao, Y., Xu, C., Xu, J., Li, H., Liu, F., Yu, D., \& Zhu, Z. (2015). The use of customized cages in revision total hip arthroplasty for Paprosky type III acetabular bone defects. International orthopaedics, 39(10), 2023-2030. https://doi.org/10.1007/s00264-015-2965-6 
Mendonça, C. R., Souza, K. T. O., Arruda, J. T., Noll, M., \& Guimarães, N. N. (2021), Human Anatomy: Teaching-Learning Experience of a Support Teacher and a Student with Low Vision and Blindness. Anatomical sciences education, 10.1002/ase.2058. https://doi.org/10.1002/ase.2058.

Miljanovic, D., Seyedmahmoudian, M., Stojcevski, A., \& Horan, B. (2020). Design and Fabrication of Implants for Mandibular and Craniofacial Defects Using Different Medical-Additive Manufacturing Technologies: A Review. Annals of biomedical engineering, 48(9), 2285-2300. https://doi.org/10.1007/s10439-020-02567-0.

Moraes, S. G., \& Muniz, A. L. (2018). Utilização de modelos 3D como recurso didático no ensino de embriologia do sistema nervoso central. Revista Da Faculdade De Ciências Médicas De Sorocaba, 20(Supl.). Recuperado de: https://revistas.pucsp.br/index.php/RFCMS/article/view/40101

Mumith, A., Thomas, M., Shah, Z., Coathup, M., \& Blunn, G. (2018). Additive manufacturing: current concepts, future trends. The bone \& joint journal, 100B(4), 455-460. https://doi.org/10.1302/0301-620X.100B4.BJJ-2017-0662.R2

Neumann, F. J., Sousa-Uva, M., Ahlsson, A., Alfonso, F., Banning, A. P., Benedetto, U., Byrne, R. A., Collet, J. P., Falk, V., Head, S. J., Jüni, P., Kastrati, A., Koller, A., Kristensen, S. D., Niebauer, J., Richter, D. J., Seferovic, P. M., Sibbing, D., Stefanini, G. G., Windecker, S., ... ESC Scientific Document Group (2019). 2018 ESC/EACTS Guidelines on myocardial revascularization. European heart journal, 40(2), 87-165. https://doi.org/10.1093/eurheartj/ehy394

Niu, G., Sapoznik, E., \& Soker, S. (2014). Bioengineered blood vessels. Expert opinion on biological therapy, 14(4), 403-410. https://doi.org/10.1517/14712598.2014.880419

Noor, N., Shapira, A., Edri, R., Gal, I., Wertheim, L., \& Dvir, T. (2019). Tissue Engineering: 3D Printing of Personalized Thick and Perfusable Cardiac Patches and Hearts (Adv. Sci. 11/2019). Advanced Science, 6(11), 1970066. https://doi.org/10.1002/advs.201970066

Provenzano, D.; Rao, Y.J.; Mitic, K.; Obaid, S.N.; Pierce, D.; Huckenpahler, J.; Berger, J.; Goyal, S.; \& Loew, M.H. (2020). Rapid Prototyping of Reusable 3D-Printed N95 Equivalent Respirators at the George Washington University. Preprints, 2020030444. doi: 10.20944/preprints202003.0444.v1

Rodrigues, V. P., Senzi Zancul, E., Gonçalves Mançanares, C., Mezzeti Giordano, C., \& Sergio Salerno, M. (2017). Additive manufacturing: state-of-the-art and application framework. G E P R O S: Gestao da Producao, Operacoes e Sistemas (Online), 12(3). https://doi.org/10.15675/gepros.v12i3.1657

Romeiro, N. C., Salomão, A., Prim, G. S., \& Vieira, M. L. H. (2019). Impressão 3D de peças anatômicas escaneadas como ferramenta de educação. Anais do $9^{\circ}$ Congresso Internacional de Design da Informação. Belo Horizonte, MG, Brasil. DOI: 10.5151/9cidi-congic-4.0337

Shakibania, S., Ghazanfari, L., Raeeszadeh-Sarmazdeh, M., \& Khakbiz, M. (2021). Medical application of biomimetic 4D printing. Drug development and industrial pharmacy, 47(4), 521-534. https://doi.org/10.1080/03639045.2020.1862179

Sheu, A. Y., Laidlaw, G. L., Fell, J. C., Triana, B. P., Goettl, C. S., \& Shah, R. P. (2019). Custom 3-Dimensional Printed Ultrasound-Compatible Vascular Access Models: Training Medical Students for Vascular Access. Journal of vascular and interventional radiology: JVIR, 30(6), 922-927. https://doi.org/10.1016/j.jvir.2019.02.011

Silva, P. C., Santandrea, R. S., Brandão, L. C., Xavier, M. V. A., \& Volpini, V. L. (2020). Manufatura aditiva: Revisão sistemática da literatura / Additive manufacturing: A systematic review. Brazilian Journal of Development, 6(11). https://doi.org/10.34117/bjdv6n11-023

Singh, R., Suri, A., Anand, S., \& Baby, B. (2016). Validation of Reverse-Engineered and Additive-Manufactured Microsurgical Instrument Prototype. Surgical innovation, 23(6), 606-612. https://doi.org/10.1177/1553350616656282

Smith, B., \& Dasgupta, P. (2020). 3D printing technology and its role in urological training. World journal of urology, 38(10), 2385-2391. https://doi.org/10.1007/s00345-019-02995-1

Smith, C. F., Tollemache, N., Covill, D., \& Johnston, M. (2018). Take away body parts! An investigation into the use of 3D-printed anatomical models in undergraduate anatomy education. Anatomical sciences education, 11(1), 44-53. https://doi.org/10.1002/ase.1718

Soares Neto, J., Barbosa, M. L. L., Matos, H. L., Xavier, A. R., Cerqueira, G. S., \& Souza, E. P. (2020). Um estudo sobre a tecnologia 3D aplicada ao ensino de anatomia: uma revisão integrativa. Research, Society and Development, 9(11), e7489119301. https://doi.org/10.33448/rsd-v9i11.9301

Su, W., Xiao, Y., He, S., Huang, P., \& Deng, X. (2018). Three-dimensional printing models in congenital heart disease education for medical students: a controlled comparative study. BMC Medical Education, 18(178), 1-6. https://doi.org/10.1186/s12909-018-1293-0

Tack, P., Victor, J., Gemmel, P., \& Annemans, L. (2016). 3D-printing techniques in a medical setting: a systematic literature review. Biomedical engineering online, 15(1), 115. https://doi.org/10.1186/s12938-016-0236-4

Taher, F., Falkensammer, J., McCarte, J., Strassegger, J., Uhlmann, M., Schuch, P., \& Assadian, A. (2017). The influence of prototype testing in threedimensional aortic models on fenestrated endograft design. Journal of vascular surgery, 65(6), 1591-1597. https://doi.org/10.1016/j.jvs.2016.10.108

Universidade Evangélica de Goiás. Catálogo de Laboratórios da UniEvangélica, Campus Rev. Arthur Wesley Archibald, 2020. Recuperado de: https://www4.unievangelica.edu.br/storage/4487/Cat\%C3\%A1logo-de-Laborat\%C3\%B3rios---2020---menor.pdf Acesso em: 16/julho/2021

Uwechue, R., Gogalniceanu, P., Kessaris, N., Byrne, N., Chandak, P., Olsburgh, J., Ahmed, K., Mamode, N., \& Loukopoulos, I. (2018). A novel 3D-printed hybrid simulation model for robotic-assisted kidney transplantation (RAKT). Journal of robotic surgery, 12(3), 541-544. https://doi.org/10.1007/s11701-0180780-y

Valverde, I. (2017). Three-dimensional Printed Cardiac Models: Applications in the Field of Medical Education, Cardiovascular Surgery, and Structural Heart Interventions. Revista espanola de cardiologia (English ed.), 70(4), 282-291. https://doi.org/10.1016/j.rec.2017.01.012. 
Research, Society and Development, v. 10, n. 12, e336101220403, 2021 (CC BY 4.0) | ISSN 2525-3409 | DOI: http://dx.doi.org/10.33448/rsd-v10i12.20403

Volpato, N., Munhoz, A. L. J., Costa, C. A., Ahrens, C. H., Carvalho, J. Santos, J. R. L., Silva, J. V. L., Foggiato, J. A., \& Lima, M. S. F. (2017). Manufatura Aditiva: Tecnologias e aplicações da impressão 3D. $1^{\text {a }}$ ed. São Paulo: Blucher.

Wang, P., Sun, Y., Shi, X., Shen, H., Ning, H., \& Liu, H. (2021a). 3D printing of tissue engineering scaffolds: a focus on vascular regeneration. Bio-design and manufacturing, 1-35. Advance online publication. https://doi.org/10.1007/s42242-020-00109-0

Wang, Y., Cui, H., Wang, Y., Xu, C., Esworthy, T. J., Hann, S. Y., Boehm, M., Shen, Y. L., Mei, D., \& Zhang, L. G. (2021b). 4D Printed Cardiac Construct with Aligned Myofibers and Adjustable Curvature for Myocardial Regeneration. ACS applied materials \& interfaces, 13(11), 12746-12758. https://doi.org/10.1021/acsami.0c17610

Wen, C. L. (2016) Homem Virtual (Ser Humano Virtual 3D): A Integração da Computação Gráfica, Impressão 3D e Realidade Virtual para Aprendizado de Anatomia, Fisiologia e Fisiopatologia. Revista de Graduação USP, 1(1), 7-15. doi: 10.11606/issn.2525-376X.v1i1p7-15.

Wilk, R., Likus, W., Hudecki, A., Syguła, M., Różycka-Nechoritis, A., \& Nechoritis, K. (2020). What would you like to print? Students' opinions on the use of 3D printing technology in medicine. PloS one, 15(4), e0230851. https://doi.org/10.1371/journal.pone.0230851.

Wu, A. M., Wang, K., Wang, J. S., Chen, C. H., Yang, X. D., Ni, W. F., \& Hu, Y. Z. (2018). The addition of 3D printed models to enhance the teaching and learning of bone spatial anatomy and fractures for undergraduate students: a randomized controlled study. Annals of translational medicine, 6(20), 403. https://doi.org/10.21037/atm.2018.09.59

Ye, Z., Dun, A., Jiang, H., Nie, C., Zhao, S., Wang, T., \& Zhai, J. (2020). The role of 3D printed models in the teaching of human anatomy: a systematic review and meta-analysis. BMC medical education, 20(1), 335. https://doi.org/10.1186/s 12909-020-02242-x 